\title{
Anaplasmataceae: Dichotomous Autophagic Interplay for Infection
}

\author{
LaNisha L. Patterson ${ }^{1}$, Caitlan D. Byerly ${ }^{1}$ and Jere W. McBride ${ }^{1,2,3,4,5^{*}}$ \\ ${ }^{1}$ Department of Pathology, University of Texas Medical Branch, Galveston, TX, United States, ${ }^{2}$ Department of Microbiology \\ and Immunology, University of Texas Medical Branch, Galveston, TX. United States, ${ }^{3}$ Center for Biodefense and Emerging \\ Infectious Diseases, University of Texas Medical Branch, Galveston, TX, United States, ${ }^{4}$ Sealy Institute for Vaccine Sciences, \\ University of Texas Medical Branch, Galveston, TX. United States, ${ }^{5}$ Institute for Human Infections and Immunity, University of \\ Texas Medical Branch, Galveston, TX, United States
}

OPEN ACCESS

Edited by:

Gee W. Lau,

University of Illinois at Urbana-

Champaign, United States

Reviewed by:

Daniel E. Voth,

University of Arkansas for Medical Sciences, United States

Eun-Kyeong Jo,

Chungnam National University,

South Korea

Ivan Tattoli,

National Institute of Health (ISS), Italy

*Correspondence:

Jere W. McBride jemcbrid@utmb.edu

Specialty section:

This article was submitted to Microbial Immunology, a section of the journal

Frontiers in Immunology

Received: 16 December 2020 Accepted: 15 March 2021 Published: 12 April 2021

Citation:

Patterson LL, Byerly CD and McBride JW (2021) Anaplasmataceae:

Dichotomous Autophagic Interplay for Infection.

Front. Immunol. 12:642771. doi: 10.3389/fimmu.2021.642771
Autophagy is a vital conserved degradative process that maintains cellular homeostasis by recycling or eliminating dysfunctional cellular organelles and proteins. More recently, autophagy has become a well-recognized host defense mechanism against intracellular pathogens through a process known as xenophagy. On the host-microbe battlefield many intracellular bacterial pathogens have developed the ability to subvert xenophagy to establish infection. Obligately intracellular bacterial pathogens of the Anaplasmataceae family, including Ehrlichia chaffeensis, Anaplasma phaogocytophilium and Orientia tsutsugamushi have developed a dichotomous strategy to exploit the host autophagic pathway to obtain nutrients while escaping lysosomal destruction for intracellular survival within the host cell. In this review, the recent findings regarding how these master manipulators engage and inhibit autophagy for infection are explored. Future investigation to understand mechanisms used by Anaplasmataceae to exploit autophagy may advance novel antimicrobial therapies and provide new insights into how intracellular microbes exploit autophagy to survive.

Keywords: autophagy, xenophagy, Anaplasmataceae, Ehrlichia, Anaplasma, effector, autolysosome, phagolysosome

\section{INTRODUCTION}

Autophagy is a well characterized host defense mechanism in which invading microbes are tagged for degradation in a selective autophagic process known as xenophagy (1-7). Although xenophagy is a known host defense mechanism against invading microbes, various intracellular pathogens including obligately intracellular rickettsial pathogens in the family Anaplasmataceae can induce autophagy as a survival mechanism $(2,5-8)$. In contrast, evasion of the autophagic pathway is also a strategy utilized by intracellular pathogens for infection. Accumulating evidence provides insight regarding the dichotomous interplay that occurs between obligately intracellular bacteria and the autophagic pathway to promote infection.

In eukaryotic cells, autophagy is a highly conserved catabolic, lysosomal-dependent process that delivers long-lived proteins and damaged cytoplasmic components to the lysosome (9-12). At basal levels, autophagy plays an important role as a response to cellular stress and maintaining homeostasis through quality control of essential cellular components. Cellular homeostasis is maintained by degrading excessive, damaged, and/or aged proteins, peptides and organelles. Macroautophagy, the best described autophagy subtype, works to sequester damaged cytoplasmic 
components in a double-membrane vesicle known as the autophagosome (13). Macroautophagy can be further categorized into nonselective autophagy which randomly engulfs cellular components within the cytoplasm into autophagosomes for degradation upon fusion with a lysosome, and selective autophagy which degrades a specific type of cargo tagged for degradation (14). Below, we will summarize the major steps of the autophagic process and the major autophagy protein groups that regulate each step of the autophagic process. Furthermore, we will discuss critical findings linking these proteins with Anaplasmataceae-induced autophagy.

The autophagic process can be divided into distinct stages, including autophagy induction, phagophore formation and elongation, cargo recognition, autophagosome maturation, lysosomal fusion and autophagosome degradation $(13,15)$. In coordination with these steps are several major signaling pathways and autophagy-related genes (ATGs). mTOR kinase is a major player in the regulation of the autophagic process $(16,17)$. Wnt and phosphoinositide 3-kinase (PI3K)/ATP dependent tyrosine kinase (Akt) signaling pathways regulate mTOR $(18,19)$. The Wnt pathway plays an essential role in inhibition of autophagy by regulating activation of the mTOR pathway. mTOR activation occurs downstream of PI3k/Akt signaling to inhibit autophagy (20, 21). Additionally, glycogen synthase kinase-3 (GSK3) inhibits the mTOR pathway by phosphorylating tuberous sclerosis complex 2 (TSC2) in a manner dependent on AMPK phosphorylation (22). Importantly, TSC2 is a Rheb GTPase-activating protein, a Ras family GTPase and an mTOR activator (23).

mTORC1 inhibition leads to autophagy induction due to activation of AMPK signaling (24). Upon decreased mTORC1 activity, the initiation of phagophore formation is stimulated by activation of the class III phosphatidylinositol 3-kinase (PtdIns3K) complex. The Ulk1 protein complex signals the formation of the PtdIns3K complex, which includes, Beclin-1 (Atg6/Vps30), Vps34 (vacuolar protein sorting 34), Vps15 (p150, a myristoylated serine/ threonine kinase), Ambra-1 (Autophagy/Beclin-1 Regulator 1), and ATG14 $(13,25,26)$. Together, the ULK1 protein complex and the PtdIns3K complex integrate nutrient status (ULK1) with autophagosome formation (PtdIns3K) (25).

Beclin-1, an orthologue of the Atg6/vacuolar protein sorting Vps30 protein in yeast, plays a central role in autophagy. Beclin-1 is important for localization of autophagic proteins to the PAS to regulate the lipid kinase Vps34 protein and promote formation of Beclin-1/Vps34/Vps15 core complexes $(13,26)$. The formation of the Beclin-1/Vps34/Vps15 complex marks the initiation of autophagy (27). The PtdIns3K complex, along with other Atg proteins, also recruits two ubiquitin-like conjugation systems, Atg12/Atg5/Atg16 and Atg8-phosphatidylethanolamine (PE), to the phagophore to recruit Atg8-PE machinery and regulate membrane elongation and expansion of the autophagosome (26, 28, 29).

Atg5/Atg12/Atg16 conjugation complex has been shown to lead to conjugation of microtubule-associated protein 1 light chain 3 (LC3), to the membrane of the autophagosome (29). This leads to the conjugation of LC3-I to phosphatidylethanolamine (PE) to form LC3-II. The p62/SQSTM1 (sequestosome 1) protein acts as a cargo receptor for ubiquitinated targets which are transported to the autophagosome for degradation $(30,31)$. Following phagophore expansion, the phagophore is completely sealed, forming the double membrane autophagosome containing all targeted components. Maturation of the autophagosome involves fusion with both early and late endosomes, which requires GTP bound small G protein Rab5, Rab7, and presenilin protein (32). The autophagosome fuses with the lysosome to form an autolysosome for degradation of engulfed components.

LC3-II and p62/SQSTM1 are also utilized as markers for autophagosome formation due to its degradation within the lysosome along with damaged and recycled components (33). Products, along with some of the autophagy cargo, are degraded by lysosomal hydrolases and recycled as amino acids supplements within the tricarboxylic acid cycle (TCA) cycle or as fatty acids, sugars, and proteins to increase energy for cell survival $(9,13)$.

\section{Autophagy as an Intracellular Innate Defense Pathway}

Although studies have demonstrated autophagy as a host defense mechanism against bacterial pathogens, many intracellular pathogens have evolved strategies to subvert autophagy for survival (34-36). Autophagy is considered a downstream effector mechanism that plays an integral role in both innate and adaptive immunity to various pathogens $(9,37)$. Xenophagy is a selective autophagy whereby intracellular pathogens are tagged by ubiquitin and subsequent targeting to autophagosomes for degradation in autolysosomes. Autophagy receptors such as p62/SQSTM1, nuclear domain 10 protein 52 (NDP52) and neighbor of BRCA1 gene 1 (NBR1) have been shown to bind ubiquitinated intracellular pathogens for autolysosome destruction and clearance (31). Autophagy plays an important role in both innate and adaptive immunity to various intracellular pathogens including Mycobacterium tuberculosis, Streptococcus pyogenes, Listeria monocytogenes, and Salmonella enterica (38-42).

This review presents the current knowledge regarding the dichotomous interplay between rickettsial pathogens in the family Anaplasmataceae, namely Ehrlichia chaffeensis, Anaplasma phaogocytophilium and Orientia tsutsugamushi, and the autophagic pathway during infection. These rickettsial pathogens utilize secreted effector proteins and host signaling pathways to hijack the autophagic pathway for survival.

\section{ANAPLASMATACEAE: INTRACELLULAR PATHOGENS OF LIFE-THREATENING HUMAN INFECTIONS}

Members of Anaplasmataceae are $\alpha$-proteobacteria in the order of Rickettsiales that include genera Anaplasma, Ehrlichia, Neorickettsia, and Orientia (43-46). Anaplasmataceae family includes obligately intracellular bacteria that reside in membrane bound cytoplasmic vacuoles mainly within 
phagocytic cells and are transmitted primarily by arthropod vectors that acquire the infection from persistently infected vertebrate hosts. These pathogens are master manipulators of the host cells (arthropod and mammalian) in which they infect. Successful intracellular infection occurs by hijacking conserved cellular signaling pathways, reprogramming host cell gene transcription, and by exploitation of other cellular processes to subvert host defense mechanisms including autophagy.

Anaplasmataceae members are best recognized for causing tick borne emerging life-threatening zoonotic diseases in the United States. Human monocytotropic ehrlichiosis (HME) and human granulocytic anaplasmosis (HGA) are group I NIAID tick-borne zoonoses caused by E. chaffeensis and $A$. phagocytophilum, respectively $(47,48)$. E. chaffeensis is maintained in nature by persistent infection of white-tailed deer, which is the primary mammalian reservoir. E. chaffeensis is transmitted by the lone star tick, Amblyomma americanum, which maintains the infection transstadially (1-3). Anaplasma phagocytophilum transstadially infects Ixodes scapularis ticks and other Ixodes spp. after acquiring the infection from infected small mammal reservoirs such as the white-footed mouse. In contrast, O. tsutsugamushi the etiologic agent of scrub typhus, a disease endemic to the Asian continent and present throughout Indonesia and northern Australia, is transmitted mainly by the bite of larva life stage-infected Leptotrombidium mites $(49,50)$. HME, HGA and scrub typhus have similar clinical presentations characterized by initial symptoms including fever, headache, myalgia, nausea, confusion, conjunctival injection (red eyes), and chills within the first two weeks following infection $(1,3,15)$. Common laboratory abnormalities include thrombocytopenia, leukopenia, anemia, and elevated hepatic transaminases (2, 14, 16-18). Disease severity ranges from mild to life-threatening complications such as toxic shock-like syndrome, kidney failure, meningoencephalitis, and acute respiratory distress $(1,2,4)$.

Members of the Anaplasmataceae family have small genomes but have evolved complex molecular strategies that enable them to create a permissive intracellular niche within professional phagocytes and other cells. Due to the obligately intracellular existence, Anaplasmataceae genomes have been shaped by a process known as reductive evolution resulting in loss of metabolic pathway genes that are no longer required for intracellular survival $(51,52)$. They replicate in membranebound cytoplasmic vacuoles within the host cell cytoplasm and undergo different developmental phases during infection. There are two well-defined ultrastructural forms, the dense-cored cell and reticulate cell, which have been identified by electron microscopy (53-56). The infectious dense-cored non replicating cell is small $(0.4-0.6 \mu \mathrm{m})$, more electron dense, and has tightly coiled nucleoid DNA. In contrast, the reticulate cell is the replicative form and is larger $(0.4-1.9 \mu \mathrm{m})$ with a dispersed nucleoid DNA. Dense-cored organisms interact with host cell receptors and enter the host cell by receptor-mediated endocytosis. After entry, dense-cored ehrlichiae transition into intermediate then full reticulate cell forms that replicate by binary fission, forming microcolonies known as morulae within host derived membrane-bound vacuoles. The ehrlichial replication cycle takes approximately $48 \mathrm{~h}$, then the replicating reticulate cells transition into infectious dense-cored ehrlichiae which are released from the host cell by cell lysis or exocytosis to infect other cells $(57,58)$.

\section{Secretion Systems and Effectors}

As with some Gram-negative bacteria, Anaplasmataceae have well known secretion systems that secrete effector proteins into the host cell. Type I, II and IV secretion systems have been identified in Anaplasmataceae. Notably, the type III secretion system found in some obligately intracellular bacteria (i.e., chlamydiae), is absent (59-61). These macromolecular secretion nanomachines are distinctly different in secretion mechanisms and the secreted effectors. Several bacterial effectors are known to regulate selective autophagy through various mechanisms for survival (62-69). Below are listed some of the secreted effector proteins by members of Anaplasmataceae that play a significant role in the subversion of autophagy.

The T1SS is well characterized in many extracellular bacteria and is known to secrete a number repeat-containing pore-forming toxins known as the Repeats in Toxin Family (RTX) $(70,71)$. The T1SS is widespread in Gram-negative bacteria and transports substrates in a one-step process across two membranes without any periplasmic intermediate into the extracellular space (72-75). Several T1SS substrates have been identified that are secreted by members of Anaplasmataceae, including ankyrin repeat (AR) and tandem repeat effector proteins. There are currently four characterized T1SS tandem repeat protein (TRP) effectors that have been identified in E. chaffeensis-infected cells including TRP32, TRP47, TRP75 and TRP120 (60, 76-78). TRPs are nucleomodulins and TRP120 has also been shown to activate host cell signaling pathways (Notch and Wnt) to downregulate innate defense mechanisms (79-82). Several of the TRPs have been shown to play a role in inhibiting TFEB nuclear localization and autolysosome generation during E. chaffeensis infection by reprogramming signal transduction pathways, including the Wnt signaling pathway (21). E. chaffeensis Ank200 is a nucleomodulin secreted by the T1SS that binds adenine-rich Alu elements in host promoter and intron regions $(83,84)$. O. tsutsugamushi secrete T1SS AR family members that traffic to diverse subcellular localizations including the endoplasmic reticulum (61). The $O$. tsutsugamushi (Ikeda strain) genome encodes 38 Ank-containing ORFs, each of which display characteristics consistent with T1SS effectors (LDAVTSIF residues found in $37-63 \%$ in their final 60 amino acids, acidic pI values and very few cysteines) (85). $O$. tsutsugamushi Anks modulate NF- $\kappa \beta$ to enhance infection; however, there is no evidence that O. tsutsugamushi T1SS substrates manipulate the autophagic pathway (86). Notably, A. phagocytophilum T1SS effectors have not been identified to date.

The T4SS is a well characterized ATP-dependent, double membrane-spanning multiprotein secretion nanomachine found in both Gram-negative and -positive bacteria (87-90). The archetypal Gram-negative T4SS is defined by the plant pathogen Agrobacterium tumefaciens VirB/D4 system (91). In Rickettsiales, a homologous but structurally different T4SS system is present with vir genes organized in three genome 
locations (92). To date, there have been a total of six T4SS effector proteins identified between E. chaffeensis and $A$. phagocytophilum; however, functions for only five of these effectors have been reported (67, 93-97). Anaplasma translocated substrate 1 (Ats-1) and ankyrin repeat domaincontaining protein A (AnkA) have been functionally characterized. Ats-1 is an orthologue of the E. chaffeensis T4SS effector protein, Etf-1. Both Ats-1 and Etf-1 play roles in subverting apoptosis and host autophagy for intracellular survival $(66,67,94,98)$. Etf-2 delays endosomal maturation to avoid routing E. chaffeensis to phagolysosomes (95). Another E. chaffeensis effector, ECH0825, is highly upregulated during early stages of infection during exponential growth in THP-1 human monocytic leukemia cells and has been shown to translocate to mitochondria where it inhibits reactive oxygen species production and host cell apoptosis by upregulating MnSOD, an essential mitochondrial antioxidant enzyme (93).

\section{PATHOGEN-HOST INTERACTIONS}

\section{E. chaffeensis}

Utilizing TRP and other effectors, E. chaffeensis avoids host immune defenses of the mononuclear phagocyte making it a remarkable model organism for examining novel host-pathogen interactions involved in cellular reprogramming. E. chaffeensis TRP effectors are secreted by the T1SS and translocate the vacuole membrane by an unknown mechanism to access the host cell. During infection, TRPs interact with a multitude of host proteins and elicit strong protective antibody responses to molecularly defined linear epitopes (99-102). TRPs are nucleomodulins that translocate to the host cell nucleus through a noncanonical NLS-independent mechanism (79-81). In addition, TRP120 has other defined functional roles during infection, and thus, is considered a moonlighting protein. These roles include promoting ehrlichial entry $(103,104)$, activation of host signaling pathways through ligand mimicry $(99,105)$, nucleomodulin activity (106-108), and as a HECT E3 ubiquitin ligase that targets host substrates for degradation $(106,107,109,110)$. The ability of E. chaffeensis to interface with the host cell is known to involve post-translation modifications including sumolyation (104), ubiqiuitination (109) and others (104). E. chaffeensis appears to exploit host cell machinery to acquire post translational modifications (PTMs) in some instances, but ehrlichial encoded ubiquitin ligases such as TRP120 are involved in creating PTMs that play a role in host-pathogen interplay (109). E. chaffeensis gene knockout studies have shown that TRP120 is essential for $E$. chaffeensis survival in vivo which can be attributed to the many defined functions of TRP120 and highlights the major role TRP120 plays in infection and survival (58).

\section{A. phagocytophilum}

A. phagocytophilum utilizes an array of bacterial proteins for adherence, invasion, and survival within the host cell. Infection is known to depend on numerous type IV secreted effector proteins, transmembrane proteins, surface proteins, and $A$. phagocytophilum-occupied vacuole membrane (AVM) proteins. These proteins include major surface protein 4 (MSP4), nucleomodulin AnkA, adhesin protein Asp14, and heat shock protein 70 (HSP70). The nucleomodulin AnkA binds host DNA and protein complexes within the nucleus of neutrophils to alter gene transcription (111). Ats-1 plays a role in preventing apoptosis by stabilizing mitochondria through the disruption of Bax-induced apoptosis to promote A. phagocytophilum infection (94).

PTMs are also involved in pathogen-host interactions. Effector protein APH0032 decorates the AVM interface and is a sumoylated by co-opting host SUMO machinery during infection. Similarly, A. phagocytophilum protein A (AmpA) is a critical effector protein that is also sumoylated to promote infection. AmpA localizes to the AVM throughout infection colocalizing with SUMO $2 / 3$ and SUMO1 as the infection progresses (112). The nucleomodulin AnkA binds host DNA and protein complexes within the nucleus of neutrophils to alter gene transcription (111).

\section{O. tsutsugamushi}

O. tsutsugamushi encodes multiple T1SS ankyrin-repeatcontaining effector proteins (Anks), known to interact with host cells and largely target the endoplasmic reticulum (113). Notably, Ank9 was the first effector shown to function during infection whereby a unique Ank9 motif mimics the GRIP domain of the host golgins, supporting O. tsutsugamushi localization to the host Golgi. Ank9 binds host protein COPB2 to hijack the endoplasmic reticulum via retrograde trafficking from the Golgi. Following its translocation, Ank9 activates the transcription factor 4-dependent unfolded protein response to support $O$. tsutsugamushi infection (114). Studies have revealed O. tsutsugamushi nucleomodulins Ank1 and Ank6 abrogate NF$\mathrm{kB}$-activated transcription utilizing exportin-1 independent mechanisms to decrease TNF $\alpha$-induced p65 nuclear levels (86).

\section{ANAPLASMATACEAE-MEDIATED EXPLOITATION OF CONSERVED HOST CELL SIGNALING PATHWAYS}

Conserved signaling pathways Wnt and Notch which play an important role in regulating innate host defenses, including phagocytosis, autophagy, and toll-like receptor (TLR) expression are exploited by $E$. chaffeensis for intracellular survival $(20,115-117)$. The $\mathrm{Wnt} / \beta$-catenin signaling pathway regulates both basal and stress-induced autophagy (20). $\beta$ catenin suppresses autophagosome formation and directly suppresses p62/SQSTM1 through T-cell factor 4 (TCF4), one of the transcriptional factors in the Wnt signaling pathway (20). Autophagy has also been shown to inhibit Notch signaling though modulation of the PTEN-PI3K/Akt/mTOR pathway (115). Inhibition of Wnt and Notch signaling dramatically reduces $E$. chaffeensis infection demonstrating the importance of the conserved cell signaling pathways for persistent infection 
and survival $(105,118)$. Notably, E. chaffeensis hijacks the canonical and non-canonical Wnt signaling pathways via effector proteins to promote infection (58). In addition, nucleomodulins TRP32, TRP47, and TRP120 bind DNA motifs within the promoter regions of Wnt target genes and may modulate Wnt gene transcription (118). Furthermore, yeast-two-hybrid $(\mathrm{Y} 2 \mathrm{H})$ analysis has identified protein-protein interactions between E. chaffeensis effector proteins (TRP32 and TRP120) and host proteins involved in Wnt signaling and transcriptional regulation of Wnt genes $(99,101)$. Of those identified, were interactions with Wnt signaling negative regulators (CEP164, KLHL12, ILF3 and LMO2) and positive regulators (PPP3R1 and VPS29) $(99,101)$.

TRP120 interaction with the host cell facilitates entry and this appears to occur via activation of non-canonical Wnt signaling resulting in $\mathrm{Ca}^{2+}$ signaling and triggering uptake through phagocytosis (118). Wnt signaling has been shown to enhance infection as RNA silencing of Wnt signaling components, including $\beta$-catenin, NFAT, CK1, and CAMKII significantly reduces $E$. chaffeensis infection, indicating that Wnt signaling is required to maintain infection (118). RNA silencing of the ubiquitously expressed Fzd5 and Fzd9 Wnt receptors, as well as the Wnt co-receptor LRP6 also results in reduced infection, indicating a possible role of the receptors for E. chaffeensis entry into the host. More specifically, RNA silencing of Fzd5 or its ligand Wnt5a results in a highly significant reduction of infection, suggesting the necessity of Wnt5a-Fzd5 signaling for E. chaffeensis entry and survival.

The Notch signaling pathway is an evolutionarily conserved pathway with critical roles in cellular homeostasis, cell proliferation and differentiation; however, Notch activation has also been shown to have significant roles in MHC class II expansion, B and T cell development, and regulation of innate immune mechanisms such as autophagy and apoptosis (119). Recently, Notch activation by $E$. chaffeensis was shown to downregulate TLR2/4 expression (105). Interestingly, TRP120 was identified as a Notch ligand mimic resulting in Notch activation as shown by nuclear translocation of the Notch intracellular domain (NICD), a hallmark for Notch activation. TRP120 is also a HECT E3 ubiquitin ligase that ubiquitinates Notch negative regulator FBW7 for proteasomal degradation resulting in increased oncoproteins levels including induced myeloid leukemia cell differentiation protein (MCL1) and NICD (107). Collectively, the data demonstrate that exploitation of conserved signaling pathways, such as Wnt and Notch is a major strategy involved in ehrlichial survival and possibly other members of the Anaplasmataceae family by modulating autophagy and other innate host defense mechanism.

\section{ANAPLASMATACEAE EFFECTOR- INDUCED AUTOPHAGY FOR NUTRIENT ACQUISITION}

Anaplasmataceae are auxotrophic with a limited capacity to synthesize required nutrients for survival, and thus, obtain essential nutrients from the host cell. Both A. phagocytophilum and $E$. chaffeensis survive by replicating within a host cell-derived membrane bound vacuole. Autophagosomes are induced by $E$. chaffeensis and A. phagocytophilum secreted T4SS effector proteins, Etf-1 and Ats-1, respectively $(67,94)$ (Figures 1 and 2). Etf-1 and Ats-1 are secreted into the host cell cytoplasm where they nucleate autophagosome formation. This effectorinduced autophagosome formation is independent of mTOR activity. Ultimately, the effector generated autophagosomes fuse with the pathogen occupied vacuoles to deliver host-derived components. Ats-1 and Etf-1 induce autophagy in a class III PtdIns3K-dependent manner and localize to inclusions with autophagosomal markers. A. phagocytophilum inclusions colocalize with early autophagosomal markers Beclin 1 and Vps34-Atg14 and are enveloped by double-lipid bilayer membranes (Figure 1) (94). Furthermore, Ats-1 directly binds to Beclin 1 and induces autophagosome formation in an ATG14 dependent manner (53); however, no interaction appears to occur with Ats-1 and UVRAG, an autophagy protein that regulates autophagosome maturation.

Studies have shown that E. chaffeensis autophagosome nucleation is dependent on Rab5-GTP and Rab5-regulated trafficking for the biogenesis of E. chaffeensis vacuoles (Figure 2) (89). Etf-1 is known to bind Rab5, Beclin 1 and phosphatidylinositol 3-kinase (PI3KC3) to induce Rab5regulated autophagy. Furthermore, E. chaffeensis ATG5 and Etf-1 were shown to localize to the membrane of inclusions and are essential for infection (54). Importantly, Etf-1 activates class III PtdIns3K, localizes with ATG5 and LC3, and interacts with RAB5-GTP, PI3CK and Beclin 1 to form a multimeric complex that fuses with E. chaffeensis inclusions. Collectively, these findings show that Etf-1 facilitates induction of RAB5-GTP autophagy through PI3CK and Beclin 1 recruitment, as well as class III PtdIns3K and ATG5 localization to E. chaffeensis inclusions. Importantly, Ehrlichia-containing vacuoles contain the late endosomal marker RAB7, as shown by mass spectrometry and confocal microscopy, but do not fuse with lysosomes (Figure 2) (120, 121).

O. tsutsugamshi induces autophagy during infection but actively escapes from autophagic destruction in dendritic cells (122). O. tsutsugamshi significantly increased endogenous LC3-II protein levels in phagocytic and nonphagocytic cells during early infection, however no significant colocalization of the bacteria and LC3-postive autophagosomes occurs $(122,123)$. Autophagy induction does not affect growth of O. tsutsugamshi as demonstrated by $3 \mathrm{MA}$ or rapamycin (autophagy inducer) treatment or use of atg3-knockout mouse embryonic fibroblasts (Atg3 $3^{-/-}$MEFs) (123). Therefore, unlike E. chaffeensis and A. phagocytophilum, O. tsutsugamshi induces autophagy; however, evades autophagosomal degradation by actively escaping from host autophagosomes. Currently, the mechanism of evasion is unknown, but is predicted to be mediated by bacterial gene expression or bacterial effector proteins.

While some pathogens hijack the autophagic pathway to replicate intracellularly, pathogens of the Anaplasmataceae 
family exploit autophagy and specific ATG proteins to acquire nutrients. However, exactly how pathogens of the Anaplasmataceae family manipulate autophagy proteins for exploitation is still unknown. Ats-1 and Etf-1 are orthologous proteins that may subvert autophagy through a similar sequence to exploit specific autophagy proteins important for nutrient acquisition (124). Additionally, some bacteria escape host autophagy through inhibition of autophagy induction. For example, S. Typhimurium inhibits autophagy initiation through regulation of the AMPKdependent activation pathway of mTOR, while M. tuberculosis inhibits autophagy induction by disruption of JNK-ROS (reactive oxygen species) signaling pathway to avoid destruction $(34,36)$. In comparison, both A. phagocytophilum and E. chaffeensis, induce autophagy independent of mTOR to acquire nutrients and remodel their vacuoles. This mechanism is regulated by T4SS effector proteins regulating key host autophagy proteins involved in the initiation step of autophagy.

\section{ANAPLASMATACEAE PREVENT ENDOSOMAL MATURATION TO AVOID LYSOSOMAL FUSION}

E. chaffeensis occupied vacuoles have features of early endosomes including RAB5, transferrin receptor (TFRC), early endosome antigen 1 (EEA1), annexins I, II, IV and VI, clathrin heavy chain and $\alpha$-adaptin $(67,121,125)$. A phagocytophilum selectively recruits Rab GTPases to avoid endosomal maturation and subsequent destruction by lysosomes (Figure 1) (126). A. phagocytophilum selectively recruits Rab GTPases that are primarily associated with recycling endosomes, including Rab4a, Rab10, Rab11A, Rab14, Rab22A and Rab35. Rab1 which mediates endoplasmic reticulum to Golgi apparatus trafficking, is also recruited to the A. phagocytophilum vacuoles $(\mathrm{ApV})$. Selectivity of Rab GTPases is shown to be dependent on A. phagocytophilum protein synthesis, allowing the ApV to disguise itself as a host recycling endosome. Importantly, the $\mathrm{ApV}$ does not mature along the endocytic pathway or resemble early endosomes due to the lack of endosomal markers including RAB5, transferrin receptor (TFRC), early endosome antigen 1 (EEA1), annexins I, II, IV and VI, clathrin heavy chain and $\alpha$ adaptin (127). Additionally, A. phagocytophilum inclusions are not acidic and do not acquire the late endosomal markers, including myeloperoxidase, CD63, LAMP-1 and V-type $\mathrm{H}+$ ATPase. Therefore, A. phagocytophilum hijacks Rab GTPases and host cell membrane traffic pathways to disguise the ApV as a recycling endosome to avoid endosomal maturation and subsequent lysosomal fusion.

Etf-2, another T4SS E. chaffeensis secreted protein effector, localizes to $E$. chaffeensis vacuoles, binds to RAB5-GTP and delays endosome maturation (Figure 2) (21). Etf-2 contains a Tre2-Bub2-Cdc16 (TBC) domain lacking Rab-GTPase activity, as well as an Arg and a Gln finger motif required for Etf-2 localization to the endosomal membrane, resulting in delayed maturation of phagosomes to phagolysosomes. EtpE is an E. chaffeensis outer membrane protein that functions as an invasion to mediate host cell entry. The C-terminal fragment of EtpE (EtpE-C) appears to be primarily responsible for E. chaffeensis binding and entry. The phagocytosis of EtpE-C-coated latex beads in Etf-2-GFP transfected cells was significantly reduced in comparison to GFP-transfected control cells. RAB5, but not RAB7, was shown to localize to a significant amount of EtpE-Ccoated latex bead containing phagosomes for a prolonged period, and no late endosomes and phagolysosomes were detected in Etf-2-GFP transfected cells, indicating delayed endosomal maturation. Etf-2 also prevents RABGAP5 localization to endosomes (95). Therefore, Etf-2 participates in blocking endosomal maturation and fusion with lysosomes to promote ehrlichial infection. Other pathogens have been shown to selectively block maturation of autophagosomes through various mechanisms, including avoidance of RAB7 recruitment $(69,128-131)$. Importantly, studies have indicated that Rab7 is essential for autophagosome maturation in general (132). Selective modulation of RAB5 function by E. chaffeensis Etf-2 leading to alterations in the autophagosome explains the selectivity in autophagosome maturation. In comparison, how the ApV recruits and hijacks specific Rab-GTPases is still unknown. Identification of A. phagocytophilum effector proteins that interact with Rab-GTPases associated with the $\mathrm{ApV}$ is critical.

Preventing lysosomal fusion is a common strategy that underlies pathogen survival. A. phagocytophilum and E. chaffeensis vacuoles fuse with autophagosomes to form intermediate organelles. E. chaffeensis intermediate organelles have been described as amphisomes (Figures 1 and 2) (21,98). Autophagosome markers Beclin 1 and LC3/GABARAP were found to colocalize with ehrlichial vacuoles indicating the fusion between autophagosomes and inclusions. Curiously, differences in LC3II localization to E. chaffeensis vacuoles have been reported. Rikihisa et al. reported no LC3II localization to ehrlichial vacuoles in RF/6A cells; however, others have detected ehrlichial vacuole localization with LC3/ GABARAP in both THP-1 and RF/6A E. chaffeensis-infected cells (95). Moreover, significant increases in LC3II levels were observed during infection and consistent with those reported for $A$. phagocytophilum. Notably, colocalization of the $A$. phagocytophilum and E. chaffeensis inclusions with lysosomal markers, including LAMP-1 and LAMP-2 were not detected. Increased p62/SQSTM1 levels were also detected in E. chaffeensisinfected cells in comparison to control cells, as another indication of inhibited lysosomal fusion. Collectively, Anaplasmataceae pathogens induce autophagy for nutrient acquisition but inhibit lysosomal maturation through selective recruitment and avoidance of specific Rab GTPases.

\section{EHRLICHIA-EXPLOITATION OF WNT SIGNALING TO INHIBIT AUTOLYSOSOME GENERATION AND AUTOPHAGIC DESTRUCTION}

Wnt and PI3k/Akt pathways are important for ehrlichial survival, and regulation of autophagy by Wnt signaling has 
been documented. E. chaffeensis utilizes TRP effectors to exploit both the Wnt and PI3k/AKT pathways to activate mTOR signaling and regulate TFEB nuclear translocation to inhibit lysosomal biogenesis and autolysosomal fusion with the pathogen occupied vacuole. E. chaffeensis activates the PI3k/ Akt pathway, a regulator of mTOR (Figure 2) (21). PI3K/Akt phosphorylates various proteins involved in regulation of cellular processes such as proliferation, apoptosis, and autophagy (133). Phosphorylated PI3K and Akt levels increase in E. chaffeensis infected cells, while phosphatase and tensin homolog (PTEN), a PI3K/Akt pathway inhibitor, levels decrease (21). The role of mTOR signaling in ehrlichial infection was also confirmed by siRNA knockdown of Rheb, a GTPase that activates mTOR. siRNA knockdown of both Rheb and phospho-p70 S6 kinase decreased E. chaffeensis infection (21). Thus, the mTOR activity is required for $E$. chaffeensis survival.

The Wnt signaling also regulates the PI3K/Akt pathway. GSK3$\beta$ is as a common protein and mediates crosstalk between PI3K/Akt and Wnt signaling pathways. More specifically, GSK3- $\beta$ regulates mTOR by induction of Tuberous Sclerosis 2 Protein (TSC2) through phosphorylation and is also a negative regulator of Wnt/ $\beta$-catenin $(22,134-136)$. Increased levels of GSK3- $\beta$ were detected in -infected cells. These effects were abrogated with treatment of a Wnt-Dvl inhibitor (21). Additionally, inhibition of Akt and induction of GSK3 resulted in a significant decrease in infected cells at early and late infection intervals. Increased levels of phospho-GSK3- $\beta$ were shown to be stimulated by T1SS effectors TRP120 and TRP32 (21). Therefore, TRP effectors activate the

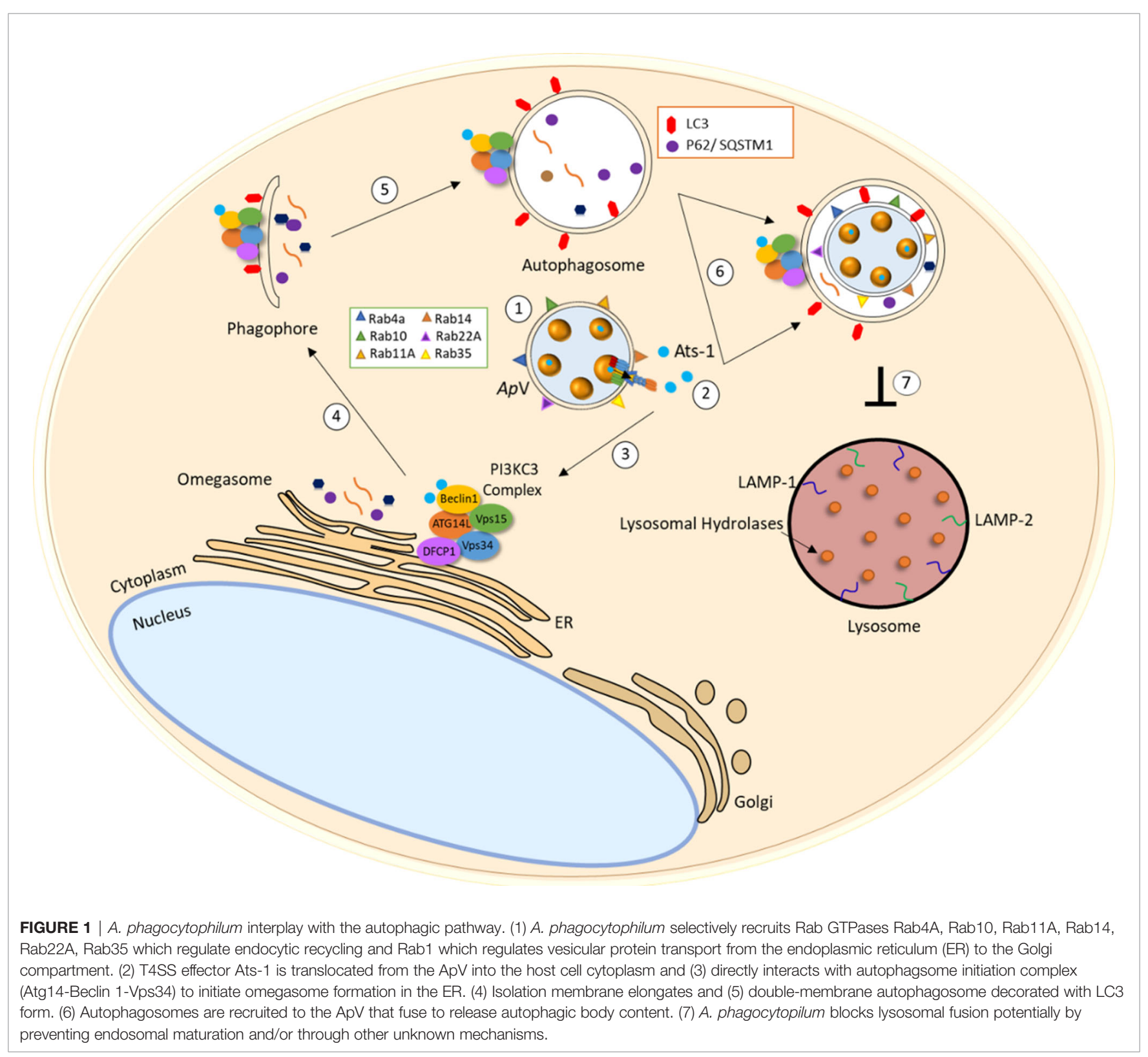




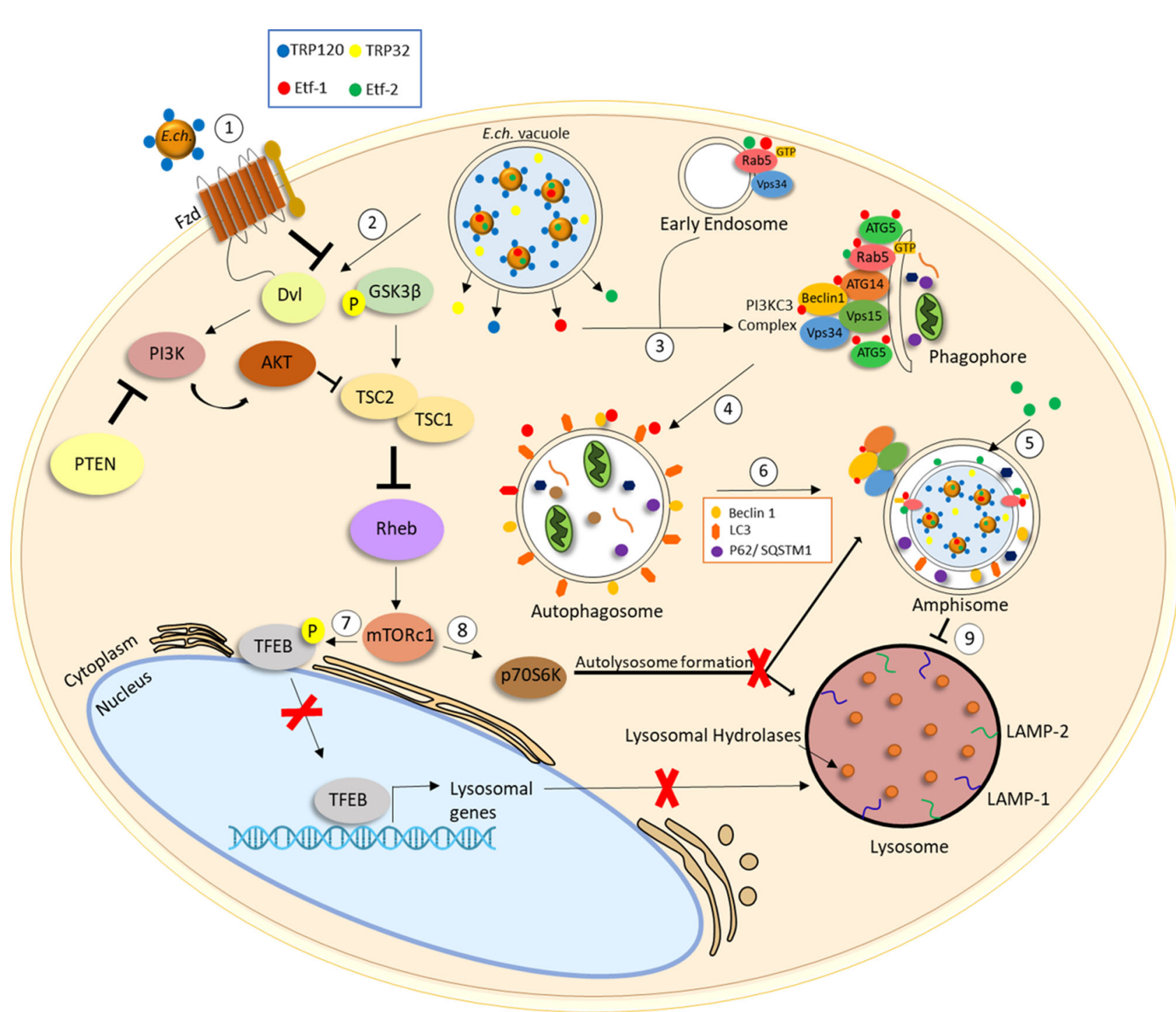

FIGURE 2 | E. chaffeensis interplay with the autophagic pathway. (1) E. chaffeensis dense-cored cells express effectors important for Wnt signaling including T1SS effectors TRP120 and TRP32. E. chaffeensis stimulates phagocytosis for entry through interaction between TRP120 and the Fzd receptor/co-receptor complex. (2) E. chaffeensis-mediated Wnt-PI3K/Akt signaling stimulates increased levels of phospho-GSK3- $\beta$ reducing TSC2 and increasing Rheb activity leading to mTOR activation. (3) E. chaffeensis T4SS effector Etf-1 is secreted into the host cell cytoplasm and interacts with Beclin1, PI3CK complex and Rab5-GTP to stimulate phagophore formation. (4) ATG5 and LC3 engage to induce autophagosome formation in a class III Ptdlns3K-dependent manner. (5) E. chaffeensis T4SS effector Etf-2 localizes to E. chaffeensis vacuole membrane and binds to RAB5-GTP to delay endosome maturation. (6) Autophagosomes displaying Beclin1, LC3II and p62/ SQSTM1 fuse with $E$. chaffeensis inclusions to form amphisomes. (7) mTOR activation leads to TFEB phosphorylation and inhibition of TFEB nuclear translocation. Inhibition of TFEB nuclear translocation prevents transcription of genes involved in lysosomal biogenesis and (8) increased phospho-p70 S6 kinase activity inhibits autolysosome formation.

PI3K/Akt pathway and inhibit GSK3 activity by phosphorylation. Decreased levels of TSC2 were also shown in E. chaffeensis-infected cells. Collectively, these findings demonstrate activation of the PI3K/Akt pathway, phosphorylation and inactivation of GSK3 and inhibition of TSC2 during E. chaffeensis infection.

Phosphorylation and inactivation of GSK3, as well as inhibition of TSC2, results in activation of $\mathrm{mTORC} 1$ and subsequent phosphorylation and inhibition of TFEB nuclear translocation. TFEB is a transcription factor that coordinates expression of lysosomal hydrolases, membrane proteins and genes involved in autophagy signaling (Figure 2). TFEB was demonstrated to remain localized in the cytoplasm during $E$. chaffeensis infection and was confirmed to be mediated by $E$. chaffeensis Wnt activation. These finding support the conclusion that E. chaffeensis exploits Wnt-PI3K-/mTOR signaling in part to regulate mTOR signaling and TFEB nuclear localization to inhibit autolysosomal generation and promote ehrlichial survival. 


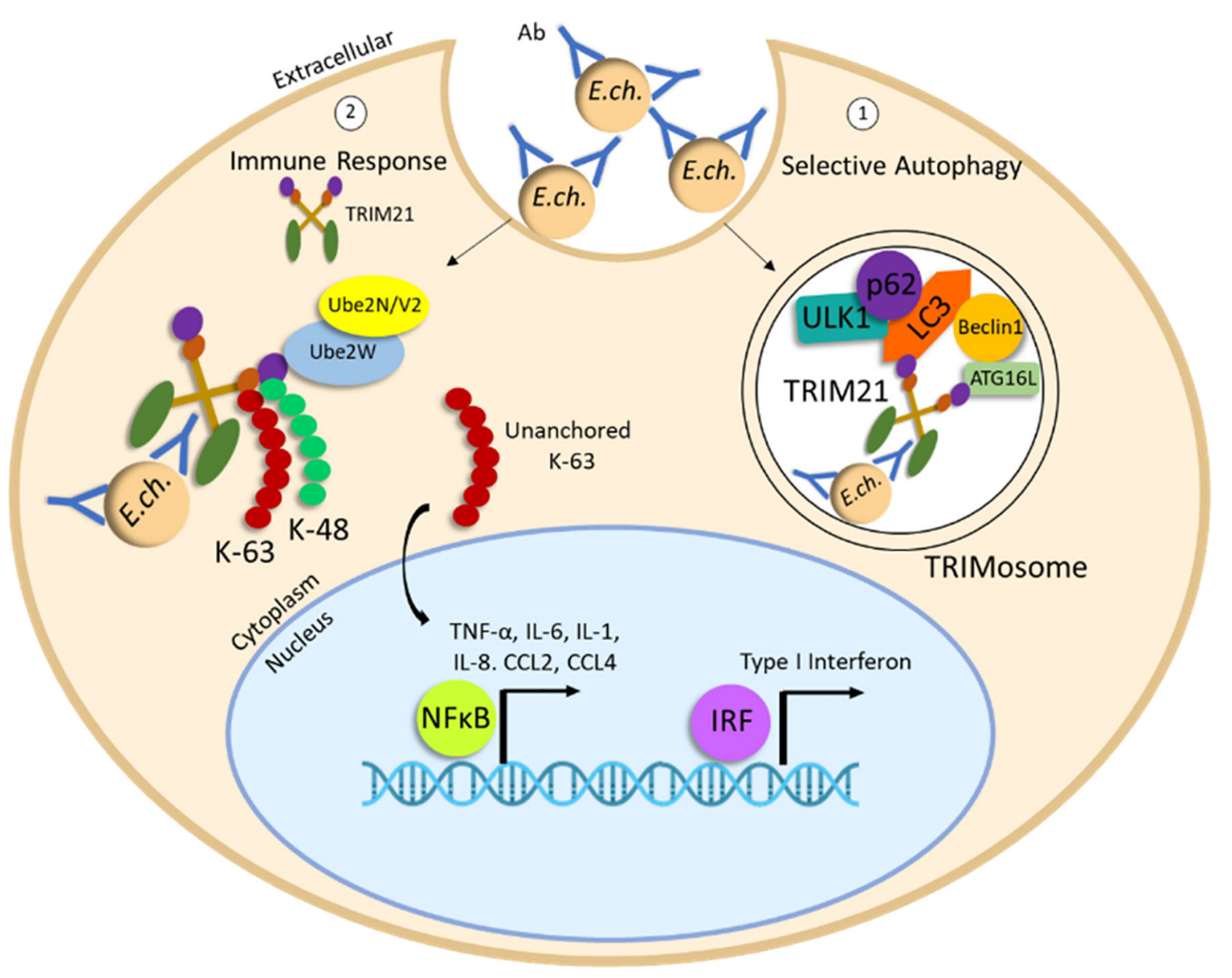

FIGURE 3 | Degradation of E. chaffeensis by antibody-TRIM21-mediated selective autophagy. Ehrlichiae opsonized with E. chaffeensis-OMP-1 specific antibody are internalized by unknown uptake mechanism. Intracellular antibody-opsonized ehrlichiae are recognized by cytosolic FC receptor TRIM21. (1) Induction of TRIMosome formation and selective autophagy occurs through recruitment of autophagy regulators, ULK1, Beclin 1, ATG16L and autophagy effectors LC3/GABARAP and p62/ SQSTM1. (2) E. chaffeensis-Ab/TRIM21 complex stimulates rapid immune signaling and a proinflammatory response through accumulation of K48 and K63 polyUb chains and activation and nuclear translocation of NF-kB and IRF.

Various studies have demonstrated the inhibition of autolysosome generation and autophagic destruction. $M$. tuberculosis inhibits Rab7 recruitment on Mtb-containing autophagosomes, while other pathogens neutralize lysosomal $\mathrm{pH}(69,131,137)$. This is the first study to elucidate the mechanism of $E$. chaffeensis inhibition of lysosomal fusion and, ultimately, destruction in the autolysosome. E. chaffeensis modulating conserved signal transduction pathways, including Wnt and Notch, to inhibit autolysosome generation may be applicable to other Anaplasmataceae bacterial pathogens.

\section{EHRLICHIA SELECTIVE AUTOPHAGIC DESTRUCTION MEDIATED BY ANTIBODY-TRIM21 COMPLEX}

Antibody-mediated immunity to E. chaffeensis is well documented involving the classical antibody Fc receptor-dependent mechanism (Figure 3). However, intracellular antibody opsonized E. chaffeensis complexes engage TRIM21, an intracellular Fc receptor (138). Antibody opsonized ehrlichiae-TRIM21 complexes recruit autophagy regulators, ULK1, Beclin 1 and autophagy effectors LC3/GABARAP and p62/SQSTM1 resulting in proinflammatory responses and localized selective autophagic degradation of the ehrlichiae-antibody complexes. These findings demonstrate the importance of autophagy engagement of adaptive immune mechanisms and provide the first example of autophagic elimination of an intracellular pathogen by a TRIM21mediated mechanism.

\section{CONCLUSIONS AND FUTURE DIRECTIONS}

Obligately intracellular pathogens of the Anaplasmataceae family have evolved highly sophisticated strategies to circumvent host immune response during infection. Autophagy is a cellular process targeted by microbial pathogens to promote infection. 
Mechanisms used by Anaplasmataceae for dichotomous engagement and subversion of autophagy for intracellular survival provides insight into the interplay that exists between the autophagic pathway and intracellular pathogens. Common amongst members of Anaplasmataceae are effector-mediated initiation of autophagy and the ability to hijack autophagy (ATG) proteins responsible for initiation and activation of the autophagic process. In contrast, effector interference with endosomal maturation contributes to pathogen survival. The ability to inhibit lysosomal destruction is a common theme demonstrated by Anaplasmataceae. This mechanism involves activation of cellular pathways such as Wnt and altering the pathogen vacuole to prevent lysosomal biogenesis and autolysosome generation. Studies to understand how Anaplasmataceae exploit the autophagic process may provide new insight; however, it is still unclear how intracellular pathogens out-compete the host for autophagy by-products and how autophagy by-products are obtained by Anaplasmataceae pathogens. It is also important to note that autophagy is an anti-inflammatory process, and it is therefore possible that Anaplasmataceae bacterial pathogens may strive to inhibit inflammation through activation of the autophagic pathway. Furthermore, very few details on the specific mechanisms that enable A. phagocytophilum and $O$. tsutsugamshi escape destruction by autophagy have been elucidated and need further investigation. Understanding mechanisms involved in autophagy induction and inhibition

\section{REFERENCES}

1. Huang J, Brumell JH. Bacteria-autophagy interplay: a battle for survival. Nat Rev Microbiol (2014) 12(2):101-14. doi: 10.1038/nrmicro3160

2. Ravenhill BJ, Boyle KB, von Muhlinen N, Ellison CJ, Masson GR, Randow F, et al. The Cargo Receptor NDP52 Initiates Selective Autophagy by Recruiting the ULK Complex to Cytosol-Invading Bacteria. Mol Cell (2019) 74(2):320-29.e6. doi: 10.1016/j.molcel.2019.01.041

3. Thurston TL, Wandel MP, von Muhlinen N, Foeglein A, Randow F. Galectin 8 targets damaged vesicles for autophagy to defend cells against bacterial invasion. Nature (2012) 482(7385):414-8. doi: 10.1038/nature10744

4. Xu Y, Jagannath C, Liu XD, Sharafkhaneh A, Kolodziejska KE, Eissa NT. Tolllike receptor 4 is a sensor for autophagy associated with innate immunity. Immunity (2007) 27(1):135-44. doi: 10.1016/j.immuni.2007.05.022

5. Yuk JM, Yoshimori T, Jo EK. Autophagy and bacterial infectious diseases. Exp Mol Med (2012) 44(2):99-108. doi: 10.3858/emm.2012.44.2.032

6. Gomes, Ligia C, Dikic I. Autophagy in Antimicrobial Immunity. Mol Cell (2014) 54(2):224-33. doi: 10.1016/j.molcel.2014.03.009

7. Mao K, Klionsky DJ. Xenophagy: A battlefield between host and microbe, and a possible avenue for cancer treatment. Autophagy (2017) 13(2):223-4. doi: 10.1080/15548627.2016.1267075

8. Mitchell G, Isberg RR. Innate Immunity to Intracellular Pathogens: Balancing Microbial Elimination and Inflammation. Cell Host Microbe (2017) 22(2):166-75. doi: 10.1016/j.chom.2017.07.005

9. Klionsky DJ, Cuervo AM, Dunn WAJr, Levine B, van der Klei I, Seglen PO. How shall I eat thee? Autophagy (2007) 3(5):413-6. doi: 10.4161/auto.4377

10. Levine B, Kroemer G. Biological Functions of Autophagy Genes: A Disease Perspective. Cell (2019) 176(1-2):11-42. doi: 10.1016/j.cell.2018.09.048

11. Mizushima N. Autophagy: process and function. Genes Dev (2007) 21 (22):2861-73. doi: 10.1101/gad.1599207

12. Mizushima N. A brief history of autophagy from cell biology to physiology and disease. Nat Cell Biol (2018) 20(5):521-7. doi: 10.1038/s41556-0180092-5 will inevitably help define how intracellular microbes exploit autophagy and could lead to novel antimicrobial therapeutic approaches.

\section{DATA AVAILABILITY STATEMENT}

The original contributions presented in the study are included in the article/supplementary material. Further inquiries can be directed to the corresponding author.

\section{AUTHOR CONTRIBUTIONS}

LP and JM conceptualized the work. LP gathered information and contributed all sections. $\mathrm{CB}$ contributed to sections pertaining to O. tsutsugamushi and exploitation of Wnt signaling. LP performed artwork. All authors contributed to the article and approved the submitted version.

\section{FUNDING}

This work was supported by the National Institutes of Health grants AI149136, AI137779, and AI123610 awarded to JM, NIH 1F31AI152424-01 fellowship to LP, and T32AI007526-20 biodefense training fellowship to $\mathrm{CB}$.

13. Nakatogawa H. Mechanisms governing autophagosome biogenesis. Nat Rev Mol Cell Biol (2020) 21(8):439-58. doi: 10.1038/s41580-020-0241-0

14. Wen X, Klionsky DJ. An overview of macroautophagy in yeast. J Mol Biol (2016) 428(9 Pt A):1681-99. doi: 10.1016/j.jmb.2016.02.021

15. Guo H, Yang Y, Feng M, Liu B, Ren X, Zhou H. Autophagy modulation in bladder cancer development and treatment (Review). Oncol Rep (2019) 42 (5):1647-55. doi: 10.3892/or.2019.7286

16. Yuan HX, Russell RC, Guan KL. Regulation of PIK3C3/VPS34 complexes by MTOR in nutrient stress-induced autophagy. Autophagy (2013) 9(12):198395. doi: 10.4161/auto. 26058

17. Ma X, Zhang S, He L, Rong Y, Brier LW, Sun Q, et al. MTORC1-mediated NRBF2 phosphorylation functions as a switch for the class III PtdIns3K and autophagy. Autophagy (2017) 13(3):592-607. doi: 10.1080/ 15548627.2016.1269988

18. Manning BD, Cantley LC. AKT/PKB signaling: navigating downstream. Cell (2007) 129(7):1261-74. doi: 10.1016/j.cell.2007.06.009

19. Ma T, Tzavaras N, Tsokas P, Landau EM, Blitzer RD. Synaptic stimulation of mTOR is mediated by Wnt signaling and regulation of glycogen synthetase kinase-3. J Neurosci (2011) 31(48):17537-46. doi: 10.1523/JNEUROSCI. 4761-11.2011

20. Petherick KJ, Williams AC, Lane JD, Ordóñez-Morán P, Huelsken J, Collard TJ, et al. Autolysosomal beta-catenin degradation regulates Wnt-autophagyp62 crosstalk. EMBO J (2013) 32(13):1903-16. doi: 10.1038/emboj.2013.123

21. Lina TT, Luo T, Velayutham TS, Das S, McBride JW. Ehrlichia activation of Wnt-PI3K-mTOR signaling inhibits autolysosome generation and autophagic destruction by the mononuclear phagocyte. Infect Immun (2017) 85(12). doi: 10.1128/IAI.00690-17

22. Inoki $\mathrm{K}$, Ouyang $\mathrm{H}$, Zhu $\mathrm{T}$, Lindvall $\mathrm{C}$, Wang $\mathrm{Y}$, Zhang $\mathrm{X}$, et al. TSC2 integrates Wnt and energy signals via a coordinated phosphorylation by AMPK and GSK3 to regulate cell growth. Cell (2006) 126(5):955-68. doi: 10.1016/j.cell.2006.06.055

23. Zhang H, Cicchetti G, Onda H, Koon HB, Asrican K, Bajraszewski N, et al. Loss of Tsc1/Tsc2 activates mTOR and disrupts PI3K-Akt signaling through 
downregulation of PDGFR. J Clin Invest (2003) 112(8):1223-33. doi: 10.1172/JCI200317222

24. Lee JW, Park S, Takahashi Y, Wang HG. The association of AMPK with ULK1 regulates autophagy. PLoS One (2010) 5(11):e15394. doi: 10.1371/ journal.pone.0015394

25. Sica V, Galluzzi L, Bravo-San Pedro JM, Izzo V, Maiuri MC, Kroemer G. Organelle-Specific Initiation of Autophagy. Mol Cell (2015) 59(4):522-39. doi: 10.1016/j.molcel.2015.07.021

26. Mizushima N, Yoshimori T, Ohsumi Y. The role of Atg proteins in autophagosome formation. Annu Rev Cell Dev Biol (2011) 27:107-32. doi: 10.1146/annurev-cellbio-092910-154005

27. Kang R, Zeh HJ, Lotze MT, Tang D. The Beclin 1 network regulates autophagy and apoptosis. Cell Death Differ (2011) 18(4):571-80. doi: 10.1038/cdd.2010.191

28. He C, Klionsky DJ. Regulation mechanisms and signaling pathways of autophagy. Annu Rev Genet (2009) 43:67-93. doi: 10.1146/annurev-genet102808-114910

29. Harada K, Kotani T, Kirisako H, Sakoh-Nakatogawa M, Oikawa Y, Kimura $\mathrm{Y}$, et al. Two distinct mechanisms target the autophagy-related E3 complex to the pre-autophagosomal structure. Elife (2019) 8. doi: 10.7554/eLife.43088

30. Pankiv S, Clausen TH, Lamark T, Brech A, Bruun JA, Outzen H, et al. p62/ SQSTM1 binds directly to Atg8/LC3 to facilitate degradation of ubiquitinated protein aggregates by autophagy. J Biol Chem (2007) 282 (33):24131-45. doi: 10.1074/jbc.M702824200

31. Tsuchiya M, Ogawa H, Koujin T, Mori C, Osakada H, Kobayashi S. p62/ SQSTM1 promotes rapid ubiquitin conjugation to target proteins after endosome rupture during xenophagy. FEBS Open Bio (2018) 8(3):470-80. doi: $10.1002 / 2211-5463.12385$

32. Berg TO, Fengsrud M, Strømhaug PE, Berg T, Seglen PO. Isolation and characterization of rat liver amphisomes. Evidence for fusion of autophagosomes with both early and late endosomes. J Biol Chem (1998) 273(34):21883-92. doi: 10.1074/jbc.273.34.21883

33. Glick D, Barth S, Macleod KF. Autophagy: cellular and molecular mechanisms. J Pathol (2010) 221(1):3-12. doi: 10.1002/path.2697

34. Ganesan R, Hos NJ, Gutierrez S, Fischer J, Stepek JM, Daglidu E, et al. Salmonella Typhimurium disrupts Sirt1/AMPK checkpoint control of mTOR to impair autophagy. PLoS Pathog (2017) 13(2):e1006227. doi: 10.1371/journal.ppat.1006227

35. Dong N, Zhu Y, Lu Q, Hu L, Zheng Y, Shao F. Structurally distinct bacterial TBC-like GAPs link Arf GTPase to Rabl inactivation to counteract host defenses. Cell (2012) 150(5):1029-41. doi: 10.1016/j.cell.2012.06.050

36. Shin DM, Jeon BY, Lee HM, Jin HS, Yuk JM, Song CH, et al. Mycobacterium tuberculosis eis regulates autophagy, inflammation, and cell death through redox-dependent signaling. PLoS Pathog (2010) 6(12):e1001230. doi: 10.1371/journal.ppat.1001230

37. Shahnazari S, Brumell JH. Mechanisms and consequences of bacterial targeting by the autophagy pathway. Curr Opin Microbiol (2011) 14 (1):68-75. doi: 10.1016/j.mib.2010.11.001

38. Deretic V, Delgado M, Vergne I, Master S, De Haro S, Ponpuak M. Autophagy in immunity against Mycobacterium tuberculosis: a model system to dissect immunological roles of autophagy. Curr Top Microbiol Immunol (2009) 335:169-88. doi: 10.1007/978-3-642-00302-8_8

39. Kimmey JM, Huynh JP, Weiss LA, Park S, Kambal A, Debnath J, et al. Unique role for ATG5 in neutrophil-mediated immunopathology during $M$. tuberculosis infection. Nature (2015) 528(7583):565-9. doi: 10.1038/ nature16451

40. Nakagawa I, Amano A, Mizushima N, Yamamoto A, Yamaguchi H, Kamimoto T, et al. Autophagy defends cells against invading group $A$ Streptococcus. Science (2004) 306(5698):1037-40. doi: 10.1126/science. 1103966

41. Birmingham CL, Canadien V, Gouin E, Troy EB, Yoshimori T, Cossart P, et al. Listeria monocytogenes evades killing by autophagy during colonization of host cells. Autophagy (2007) 3(5):442-51. doi: 10.4161/auto.4450

42. Wu S, Shen Y, Zhang S, Xiao Y, Shi S. Salmonella Interacts With Autophagy to Offense or Defense. Front Microbiol (2020) 11:721. doi: 10.3389/ fmicb.2020.00721

43. Min CK, Yang JS, Kim S, Choi MS, Kim IS, Cho NH. Genome-based construction of the metabolic pathways of Orientia tsutsugamushi and comparative analysis within the Rickettsiales order. Comp Funct Genomics (2008) 2008:623145. doi: 10.1155/2008/623145

44. Dumler JS, Barbet AF, Bekker CP, Dasch GA, Palmer GH, Ray SC, et al. Reorganization of genera in the families Rickettsiaceae and Anaplasmataceae in the order Rickettsiales: unification of some species of Ehrlichia with Anaplasma, Cowdria with Ehrlichia and Ehrlichia with Neorickettsia, descriptions of six new species combinations and designation of Ehrlichia equi and 'HGE agent' as subjective synonyms of Ehrlichia phagocytophila. Int J Syst Evol Microbiol (2001) 51(Pt 6):2145-65. doi: 10.1099/00207713-516-2145

45. Pruneau L, Lebrigand K, Mari B, Lefrançois T, Meyer DF, Vachiery N. Understanding Anaplasmataceae pathogenesis using "Omics" approaches. Front Cell Infect Microbiol (2014) 4:86. doi: 10.3389/fcimb.2014.00086

46. McBride JW, Walker DH. Molecular and cellular pathobiology of Ehrlichia infection: targets for new therapeutics and immunomodulation strategies. Expert Rev Mol Med (2011) 13:e3. doi: 10.1017/S1462399410001730

47. Anderson BE, Sumner JW, Dawson JE, Tzianabos T, Greene CR, Olson JG, et al. Detection of the etiologic agent of human ehrlichiosis by polymerase chain reaction. J Clin Microbiol (1992) 30(4):775-80. doi: 10.1128/ JCM.30.4.775-780.1992

48. Chen SM, Dumler JS, Bakken JS, Wlaker DH. Identification of a granulocytotropic Ehrlichia species as the etiologic agent of human disease. J Clin Microbiol (1994) 32(3):589-95. doi: 10.1128/JCM.32.3.589-595.1994

49. Paris DH, Shelite TR, Day NP, Walker DH. Unresolved problems related to scrub typhus: a seriously neglected life-threatening disease. Am J Trop Med Hyg (2013) 89(2):301-7. doi: 10.4269/ajtmh.13-0064

50. Valbuena G, Walker DH. Approaches to vaccines against Orientia tsutsugamushi. Front Cell Infect Microbiol (2012) 2:170. doi: 10.3389/ fcimb.2012.00170

51. Dunning Hotopp JC, Lin M, Madupu R, Crabtree J, Angiuoli SV, Eisen JA, et al. Comparative genomics of emerging human ehrlichiosis agents. PLoS Genet (2006) 2(2):e21. doi: 10.1371/journal.pgen.0020021

52. Andersson SG, Kurland CG. Reductive evolution of resident genomes. Trends Microbiol (1998) 6(7):263-8. doi: 10.1016/S0966-842X(98)01312-2

53. Munderloh UG, Jauron SD, Fingerle V, Leitritz L, Hayes SF, Hautman JM, et al. Invasion and intracellular development of the human granulocytic ehrlichiosis agent in tick cell culture. J Clin Microbiol (1999) 37(8):2518-24. doi: 10.1128/JCM.37.8.2518-2524.1999

54. Popov VL, Han VC, Chen SM, Dumler JS, Feng HM, Andreadis TG, et al. Ultrastructural differentiation of the genogroups in the genus Ehrlichia. J. Med. Microbiol (1998) 47(3):235-51. doi: 10.1099/00222615-47-3-235

55. Troese MJ. Anaplasma phagocytophilum dense-cored organisms mediate cellular adherence through recognition of human P-selectin glycoprotein ligand 1. Infect Immun (2009) 77(9):4018-27. doi: 10.1128/IAI.00527-09

56. Frutos R, Viari A, Vachiery N, Boyer F, Martinez D. Ehrlichia ruminantium: genomic and evolutionary features. Trends Parasitol (2007) 23(9):414-9. doi: 10.1016/j.pt.2007.07.007

57. Paddock CD, Childs JE. Ehrlichia chaffeensis: a prototypical emerging pathogen. Clin Microbiol Rev (2003) 16(1):37-64. doi: doi: 10.1128/ cmr.16.1.37-64.2003

58. Lina TT, Farris T, Luo T, Mitra S, Zhu B, McBride JW. Hacker within! Ehrlichia chaffeensis effector driven phagocyte reprogramming strategy. Front Cell Infect Microbiol (2016) 6:58. doi: doi: 10.3389/fcimb.2016.00058

59. Al-Khedery B, Lundgren AM, Stuen S, Granquist EG, Munderloh UG, Nelson CM, et al. Structure of the type IV secretion system in different strains of Anaplasma phagocytophilum. BMC Genomics (2012) 13(1):678. doi: 10.1186/1471-2164-13-678

60. Wakeel A, den Dulk-Ras A, Hooykaas PJ, McBride JW. Ehrlichia chaffeensis tandem repeat proteins and Ank200 are type 1 secretion system substrates related to the repeats-in-toxin exoprotein family. Front Cell Infect Microbiol (2011) 1:22. doi: doi: 10.3389/fcimb.2011.00022

61. VieBrock L, Evans SM, Beyer AR, Larson CL, Beare PA, Ge H, et al. Orientia tsutsugamushi ankyrin repeat-containing protein family members are Type 1 secretion system substrates that traffic to the host cell endoplasmic reticulum. Front Cell Infect Microbiol (2014) 4:186. doi: 10.3389/ fcimb.2014.00186

62. Choy A, Dancourt J, Mugo B, O'Connor TJ, Isberg RR, Melia TJ. The Legionella effector RavZ inhibits host autophagy through irreversible Atg8 
deconjugation. Science (2012) 338(6110):1072-6. doi: 10.1126/ science. 1227026

63. Itakura E, Kishi-Itakura C, Mizushima N. The hairpin-type tail-anchored SNARE syntaxin 17 targets to autophagosomes for fusion with endosomes/ lysosomes. Cell (2012) 151(6):1256-69. doi: 10.1016/j.cell.2012.11.001

64. Matsushima N, Yoshida H, Kumaki Y, Kamiya M, Tanaka T, et al. Flexible structures and ligand interactions of tandem repeats consisting of proline, glycine, asparagine, serine, and/or threonine rich oligopeptides in proteins. Curr Protein Pept Sci (2008) 9(6):591-610. doi: 10.2174/1389203 08786733886

65. Li R, Tan S, Yu M, Jundt MC, Zhang S, Wu M. Annexin A2 Regulates Autophagy in Pseudomonas aeruginosa Infection through the Akt1-mTORULK1/2 Signaling Pathway. J Immunol (2015) 195(8):3901-11. doi: 10.4049/ jimmunol.1500967

66. Niu H, Rikihisa Y. Ats-1: a novel bacterial molecule that links autophagy to bacterial nutrition. Autophagy (2013) 9(5):787-8. doi: 10.4161/auto.23693

67. Lin M, Liu H, Xiong Q, Niu H, Cheng Z, Yamamoto A. Ehrlichia secretes Etf-1 to induce autophagy and capture nutrients for its growth through RAB5 and class III phosphatidylinositol 3-kinase. Autophagy (2016) 12 (11):2145-66. doi: 10.1080/15548627.2016.1217369

68. Yoshikawa Y, Ogawa M, Hain T, Yoshida M, Fukumatsu M, Kim M, et al. Listeria monocytogenes ActA-mediated escape from autophagic recognition. Nat Cell Biol (2009) 11(10):1233-40. doi: 10.1038/ncb1967

69. Chandra P, Ghanwat S, Matta SK, Yadav SS, Mehta M, Siddiqui Z, et al. Mycobacterium tuberculosis Inhibits RAB7 Recruitment to Selectively Modulate Autophagy Flux in Macrophages. Sci Rep (2015) 5:16320. doi: 10.1038/srep16320

70. Bumba L, Masin J, Macek P, Wald T, Motlova L, Bibova I, et al. CalciumDriven Folding of RTX Domain beta-Rolls Ratchets Translocation of RTX Proteins through Type I Secretion Ducts. Mol Cell (2016) 62(1):47-62. doi: 10.1016/j.molcel.2016.03.018

71. Thomas S, Holland IB, Schmitt L. The Type 1 secretion pathway - The hemolysin system and beyond. Biochim Biophys Acta (2013) 1843(8):162941. doi: 10.1016/j.bbamcr.2013.09.017

72. Green ER, Mecsas J. Bacterial Secretion Systems: An Overview. Microbiol Spectr (2016) 4(1). doi:10.1128/microbiolspec.VMBF-0012-2015

73. Spitz O, Erenburg IN, Beer T, Kanonenberg K, Holland IB, Schmitt L. Type I Secretion Systems-One Mechanism for All? Microbiol Spectr (2019) 7(2). doi: 10.1128/microbiolspec.PSIB-0003-2018

74. Holland IB, Schmitt L, Young J. Type 1 protein secretion in bacteria, the ABC-transporter dependent pathway (review). Mol Membr Biol (2005) 22(12):29-39. doi:10.1080/09687860500042013

75. Delepelaire P. Type I secretion in gram-negative bacteria. Biochim Biophys Acta (2004) 1694(1-3):149-61. doi:10.1016/j.bbamcr.2004.05.001

76. Luo T, Zhang X, Wakeel A, Popov VL, McBride JW. A variable-length PCR target protein of Ehrlichia chaffeensis contains major species-specific antibody epitopes in acidic serine-rich tandem repeats. Infect Immun (2008) 76(4):1572-80. doi: 10.1128/IAI.01466-07

77. Luo T, Zhang X, McBride JW. Major species-specific antibody epitopes of the Ehrlichia chaffeensis p120 and E. canis p140 orthologs in surface-exposed tandem repeat regions. Clin Vaccine Immunol (2009) 16(7):982-90. doi: 10.1128/CVI.00048-09

78. Luo T, Zhang X, Nicholson WL, Zhu B, McBride JW, et al. Molecular characterization of antibody epitopes of Ehrlichia chaffeensis ankyrin protein 200 and tandem repeat protein 47 and evaluation of synthetic immunodeterminants for serodiagnosis of human monocytotropic ehrlichiosis. Clin Vaccine Immunol (2010) 17(1):87-97. doi: 10.1128/ CVI.00331-09

79. Klema VJ, Sepuru KM, Füllbrunn N, Farris TR, Dunphy PS, McBride JW, et al. Ehrlichia chaffeensis TRP120 nucleomodulin binds DNA with disordered tandem repeat domain. PLoS One (2018) 13(4):e0194891. doi: 10.1371/journal.pone.0194891

80. Farris TR, Dunphy PS, Zhu B, Kibler CE, McBride JW. Ehrlichia chaffeensis TRP32 is a nucleomodulin that directly regulates expression of host genes governing differentiation and proliferation. Infect Immun (2016) 84 (11):3182-94. doi: 10.1128/IAI.00657-16

81. Kibler CE, Milligan SL, Farris TR, Zhu B, Mitra S, McBride JW. Ehrlichia chaffeensis TRP47 enters the nucleus via a MYND-binding domain- dependent mechanism and predominantly binds enhancers of host genes associated with signal transduction, cytoskeletal organization, and immune response. PLoS One (2018) 13(11):e0205983. doi: 10.1371/journal.pone. 0205983

82. Dunphy PS, Luo T, McBride JW. Ehrlichia moonlighting effectors and interkingdom interactions with the mononuclear phagocyte. Microbes Infect (2013) 15(14-15):1005-16. doi: 10.1016/j.micinf.2013.09.011

83. Wakeel A, Zhang X, McBride JW. Mass spectrometric analysis of Ehrlichia chaffeensis tandem repeat proteins reveals evidence of phosphorylation and absence of glycosylation. PLoS One (2010) 5(3):e9552. doi: 10.1371/ journal.pone.0009552

84. Zhu B, Nethery KA, Kuriakose JA, Wakeel A, Zhang X, McBride JW. Nuclear translocated Ehrlichia chaffeensis ankyrin protein interacts with a specific adenine-rich motif of host promoter and intronic Alu elements. Infect Immun (2009) 77(10):4243-55. doi: 10.1128/IAI.00376-09

85. Beyer AR, VieBrock L, Rodino KG, Miller DP, Tegels BK, Marconi RT, Carlyon JA. Orientia tsutsugamushi Strain Ikeda Ankyrin Repeat-Containing Proteins Recruit SCF1 Ubiquitin Ligase Machinery via Poxvirus-Like F-Box Motifs. J Bacteriol (2015) 197(19):3097-109. doi: 10.1128/JB.00276-15

86. Evans SM, Rodino KG, Adcox HE, Carlyon JA. Orientia tsutsugamushi uses two Ank effectors to modulate NF-kappaB p65 nuclear transport and inhibit NF-kappaB transcriptional activation. PLoS Pathog (2018) 14(5):e1007023. doi: 10.1371/journal.ppat.1007023

87. Fronzes R, Christie PJ, Waksman G. The structural biology of type IV secretion systems. Nat Rev Microbiol (2009) 7(10):703-14. doi: 10.1038/ nrmicro2218

88. Berge C, Waksman G, Terradot L. Structural and Molecular Biology of Type IV Secretion Systems. Curr Top Microbiol Immunol (2017) 413:31-60. doi: 10.1007/978-3-319-75241-9_2

89. Chandran Darbari V, Waksman G. Structural Biology of Bacterial Type IV Secretion Systems. Annu Rev Biochem (2015) 84:603-29. doi: 10.1146/ annurev-biochem-062911-102821

90. Fronzes R, Schäfer E, Wang L, Saibil HR, Orlova EV, Waksman G. Structure of a type IV secretion system core complex. Science (2009) 323(5911):266-8. doi: $10.1126 /$ science.1166101

91. Bundock P, Meaney RS, Hamadache S, Janakirama P, Edgell DR, Karas BJ. Trans-kingdom T-DNA transfer from Agrobacterium tumefaciens to Saccharomyces cerevisiae. EMBO J (1995) 14(13):3206-14. doi: 10.1002/ j.1460-2075.1995.tb07323.x

92. Gillespie JJ, Ammerman NC, Dreher-Lesnick SM, Rahman MS, Worley MJ, Setubal JC, et al. An anomalous type IV secretion system in Rickettsia is evolutionarily conserved. PLoS One (2009) 4(3):e4833. doi: 10.1371/ journal.pone. 0004833

93. Liu H, Bao W, Lin M, Niu H, Rikihisa Y. Ehrlichia type IV secretion effector ECH0825 is translocated to mitochondria and curbs ROS and apoptosis by upregulating host MnSOD. Cell Microbiol (2012) 14(7):1037-50. doi: 10.1111/j.1462-5822.2012.01775.x

94. Niu H, Kozjak-Pavlovic V, Rudel T, Rikihisa Y. Anaplasma phagocytophilum Ats-1 is imported into host cell mitochondria and interferes with apoptosis induction. PLoS Pathog (2010) 6(2):e1000774. doi: 10.1371/ journal.ppat.1000774

95. Yan Q, Lin M, Huang W, Teymournejad O, Johnson JM, Hays FA, et al. Ehrlichia type IV secretion system effector Etf-2 binds to active RAB5 and delays endosome maturation. Proc Natl Acad Sci U S A (2018) 115(38): E8977-86. doi: 10.1073/pnas.1806904115

96. Caturegli P, Asanovich KM, Walls JJ, Bakken JS, Madigan JE, Popov VL, Dumler JS. ankA: an Ehrlichia phagocytophila group gene encoding a cytoplasmic protein antigen with ankyrin repeats. Infect Immun (2000) 68 (9):5277-83. doi: 10.1128/iai.68.9.5277-5283.2000

97. Sinclair SH, Garcia-Garcia JC, Dumler JS. Bioinformatic and mass spectrometry identification of Anaplasma phagocytophilum proteins translocated into host cell nuclei. Front Microbiol (2015) 6:55. doi: 10.3389/fmicb.2015.00055

98. Rikihisa Y. Subversion of RAB5-regulated autophagy by the intracellular pathogen Ehrlichia chaffeensis. Small GTPases (2017) p:1-7. doi: 10.1080/ 21541248.2017.1332506

99. Luo T, Kuriakose JA, Zhu B, Wakeel A, McBride JW. Ehrlichia chaffeensis TRP120 interacts with a diverse array of eukaryotic proteins involved in 
transcription, signaling, and cytoskeleton organization. Infect Immun (2011) 79(11):4382-91. doi: 10.1128/IAI.05608-11

100. Wakeel A, Kuriakose JA, McBride JW. An Ehrlichia chaffeensis tandem repeat protein interacts with multiple host targets involved in cell signaling, transcriptional regulation, and vesicle trafficking. Infect Immun (2009) 77 (5):1734-45.

101. Luo T, McBride JW. Ehrlichia chaffeensis TRP32 interacts with host cell targets that influence intracellular survival. Infect Immun (2012) 80(7):2297306. doi: 10.1128/IAI.00154-12

102. Luo T, Mitra S, McBride JW. Ehrlichia chaffeensis TRP75 Interacts with host cell targets involved in homeostasis, cytoskeleton organization, and apoptosis regulation to promote infection. mSphere (2018) 3(2):e00147-18. doi: 10.1128/mSphere.00147-18

103. Popov VL, Yu X, Walker DH. The $120 \mathrm{kDa}$ outer membrane protein of Ehrlichia chaffeensis: preferential expression on dense-core cells and gene expression in Escherichia coli associated with attachment and entry. Microb Pathog (2000) 28(2):71-80. doi: 10.1006/mpat.1999.0327

104. Dunphy PS, Luo T, McBride JW. Ehrlichia chaffeensis exploits host SUMOylation pathways to mediate effector-host interactions and promote intracellular survival. Infect Immun (2014) 82(10):4154-68. doi: 10.1128/ IAI.01984-14

105. Lina TT, Dunphy PS, Luo T, McBride JW. Ehrlichia chaffeensis TRP120 activates canonical Notch signaling to downregulate TLR2/4 expression and promote intracellular survival. MBio (2016) 7(4). doi: 10.1128/mBio.0067216

106. Mitra S, Dunphy PS, Das S, Zhu B, Luo T, McBride JW. Ehrlichia chaffeensis TRP120 effector targets and recruits host polycomb group proteins for degradation to promote intracellular infection. Infect Immun (2018) 86(4). doi: 10.1128/IAI.00845-17

107. Wang JY, Zhu B, Patterson LL, Rogan MR, Kibler CE, McBride JW. Ehrlichia chaffeensis TRP120-mediated ubiquitination and proteasomal degradation of tumor suppressor FBW7 increases oncoprotein stability and promotes infection. PLoS Pathog (2020) 16(4):e1008541. doi: 10.1371/ journal.ppat.1008541

108. Zhu B, Kuriakose JA, Luo T, Ballesteros E, Gupta S, Fofanov Y, McBride JW. Ehrlichia chaffeensis TRP120 binds a G+C-rich motif in host cell DNA and exhibits eukaryotic transcriptional activator function. Infect Immun (2011) 79(11):4370-81. doi: 10.1128/IAI.05422-11

109. Zhu B, Das S, Mitra S, Farris TR, McBride JW. Ehrlichia chaffeensis TRP120 moonlights as a HECT E3 ligase involved in self and host ubiquitination to influence protein interactions and stability for intracellular survival. Infect Immun (2017). doi: 10.1128/IAI.00290-17

110. Zhu B, Farris TR, Milligan SL, Chen H, Zhu R, Hong A, et al. Rapid identification of ubiquitination and sumoylation target sites by microfluidic peptide array. Biochem Biophysical Res Comm (2015) 5:430-8. doi: 10.1016/ j.bbrep.2016.02.003

111. Park J, Kim KJ, Choi KS, Grab DJ, Dumler JS. Anaplasma phagocytophilum AnkA binds to granulocyte DNA and nuclear proteins. Cell Microbiol (2004) 6(8):743-51. doi: 10.1111/j.1462-5822.2004.00400.x

112. Beyer AR, Truchan HK, May LJ, Walker NJ, Borjesson DL, Carlyon JA. The Anaplasma phagocytophilum effector AmpA hijacks host cell SUMOylation. Cell Microbiol (2015) 17(4):504-19. doi: 10.1111/ cmi. 12380

113. Wongsantichon J, Jaiyen Y, Dittrich S, Salje J. Orientia tsutsugamushi. Trends Microbiol (2020) 28(9):780-1. doi: 10.1016/j.tim.2020.02.014

114. Beyer AR, Rodino KG, VieBrock L, Green RS, Tegels BK, Oliver LD Jr, et al. Orientia tsutsugamushi Ank9 is a multifunctional effector that utilizes a novel GRIP-like Golgi localization domain for Golgi-to-endoplasmic reticulum trafficking and interacts with host COPB2. Cell Microbiol (2017) 19(7). doi: $10.1111 / \mathrm{cmi} .12727$

115. Song BQ, Chi Y, LiX, Du WJ, Han ZB, Tian JJ, et al. Inhibition of Notch Signaling Promotes the Adipogenic Differentiation of Mesenchymal Stem Cells Through Autophagy Activation and PTEN-PI3K/AKT/mTOR Pathway. Cell Physiol Biochem (2015) 36(5):1991-2002. doi: 10.1159/000430167

116. Hu X, Chung AY, Wu I, Foldi J, Chen J, Ji JD, et al. Integrated regulation of Toll-like receptor responses by Notch and interferon-gamma pathways. Immunity (2008) 29(5):691-703. doi: 10.1016/j.immuni.2008.08.016
117. Maiti G, Naskar D, Sen M. The Wingless homolog Wnt5a stimulates phagocytosis but not bacterial killing. Proc Natl Acad Sci U S A (2012) 109 (41):16600-5. doi: 10.1073/pnas.1207789109

118. Luo T, Dunphy PS, Lina TT, McBride. Ehrlichia chaffeensis exploits canonical and noncanonical host Wnt signaling pathways to stimulate phagocytosis and promote intracellular survival. Infect Immun (2015) 84 (3):686-700. doi: 10.1128/IAI.01289-15

119. Palaga T, Ratanabunyong S, Pattarakankul T, Sangphech N, Wongchana W, Hadae $\mathrm{Y}$, Kueanjinda P. Notch signaling regulates expression of Mcl-1 and apoptosis in PPD-treated macrophages. Cell Mol Immunol (2013) 10(5):44452. doi: $10.1038 / \mathrm{cmi} .2013 .22$

120. Cheng Y, Liu Y, Wu B, Zhang JZ, Gu J, Liao YL, et al. Proteomic analysis of the Ehrlichia chaffeensis phagosome in cultured DH82 cells. PLoS One (2014) 9(2):e88461. doi: 10.1371/journal.pone.0088461

121. Rikihisa Y. Molecular Pathogenesis of Ehrlichia chaffeensis Infection. Annu Rev Microbiol (2015) 69:283-304. doi: 10.1146/annurev-micro-091014104411

122. Choi JH, Cheong TC, Ha NY, Ko Y, Cho CH, Jeon JH, et al. Orientia tsutsugamushi subverts dendritic cell functions by escaping from autophagy and impairing their migration. PLoS Negl Trop Dis (2013) 7(1):e1981. doi: 10.1371/journal.pntd.0001981

123. Ko Y, Choi JH, Ha NY, Kim IS, Cho NH, Choi MS. Active escape of Orientia tsutsugamushi from cellular autophagy. Infect Immun (2013) 81(2):552-9. doi: 10.1128/IAI.00861-12

124. Rikihisa Y. Role and function of the Type IV secretion system in Anaplasma and Ehrlichia species. Curr Top Microbiol Immunol (2017) 413:297-321. doi: 10.1007/978-3-319-75241-9_12

125. Barnewall RE, Rikihisa Y, Lee EH. Ehrlichia chaffeensis inclusions are early endosomes which selectively accumulate transferrin receptor. Infect Immun (1997) 65(4):1455-61. doi: 10.1128/IAI.65.4.1455-1461.1997

126. Huang B, Hubber A, McDonough JA, Roy CR, Scidmore MA, Carlyon JA. The Anaplasma phagocytophilum-occupied vacuole selectively recruits Rab-GTPases that are predominantly associated with recycling endosomes. Cell Microbiol (2010) 12(9):1292-307. doi: 10.1111/j.14625822.2010.01468.x

127. Mott J, Barnewall RE, Rikihisa Y. Human granulocytic ehrlichiosis agent and Ehrlichia chaffeensis reside in different cytoplasmic compartments in HL60 cells. Infect Immun (1999) 67(3):1368-78. doi: 10.1128/IAI.67.3.13681378.1999

128. Seto S, Matsumoto S, Tsujimura K, Koide Y. Differential recruitment of CD63 and Rab7-interacting-lysosomal-protein to phagosomes containing Mycobacterium tuberculosis in macrophages. Microbiol Immunol (2010) 54 (3):170-4. doi: 10.1111/j.1348-0421.2010.00199.x

129. Kumar R, Sahu SK, Kumar M, Jana K, Gupta P, Gupta UD, et al. MicroRNA 17-5p regulates autophagy in Mycobacterium tuberculosis-infected macrophages by targeting Mcl-1 and STAT3. Cell Microbiol (2016) 18 (5):679-91. doi: $10.1111 / \mathrm{cmi} .12540$

130. Yasir M, Pachikara ND, Bao X, Pan Z, Fan H. Regulation of chlamydial infection by host autophagy and vacuolar ATPase-bearing organelles. Infect Immun (2011) 79(10):4019-28. doi: 10.1128/IAI.05308-11

131. Pujol C, Klein KA, Romanov GA, Palmer LE, Cirota C, Zhao Z, Bliska JB. Yersinia pestis can reside in autophagosomes and avoid xenophagy in murine macrophages by preventing vacuole acidification. Infect Immun (2009) 77 (6):2251-61. doi: 10.1128/IAI.00068-09

132. Jager S, Bucci C, Tanida I, Ueno T, Kominami E, Saftig P, Eskelinen EL. Role for Rab7 in maturation of late autophagic vacuoles. J Cell Sci (2004) 117(Pt 20):4837-48. doi: 10.1242/jcs. 01370

133. Yu JS, Cui W. Proliferation, survival and metabolism: the role of PI3K/AKT/ mTOR signalling in pluripotency and cell fate determination. Development (2016) 143(17):3050-60. doi: 10.1242/dev.137075

134. Zhang HH, Lipovsky AI, Dibble CC, Sahin M, Manning BD. S6K1 regulates GSK3 under conditions of mTOR-dependent feedback inhibition of Akt. Mol Cell (2006) 24(2):185-97. doi: 10.1016/j.molcel.2006.09.019

135. Vigneron F, Dos Santos P, Lemoine S, Bonnet M, Tariosse L, Couffinhal T, Duplaà C, Jaspard-Vinassa B. GSK-3beta at the crossroads in the signalling of heart preconditioning: implication of mTOR and Wnt pathways. Cardiovasc Res (2011) 90(1):49-56. doi: 10.1093/cvr/cvr002 
136. Inoki $\mathrm{K}$, Li Y, Zhu T, Wu J, Guan KL. TSC2 is phosphorylated and inhibited by Akt and suppresses mTOR signalling. Nat Cell Biol (2002) 4 (9):648-57. doi: $10.1038 / \mathrm{ncb} 839$

137. Miao Y, Li G, Zhang X, Xu H, Abraham SN. A TRP Channel Senses Lysosome Neutralization by Pathogens to Trigger Their Expulsion. Cell (2015) 161(6):1306-19. doi: 10.1016/j.cell.2015.05.009

138. Velayutham TS, Kumar S, Zhang X, Kose N, Walker DH, Winslow G, Crowe JE Jr, McBride JW. Ehrlichia chaffeensis Outer Membrane Protein 1-Specific Human Antibody-Mediated Immunity Is Defined by Intracellular TRIM21Dependent Innate Immune Activation and Extracellular Neutralization. Infect Immun (2019) 87(12). doi: 10.1128/IAI.00383-19
Conflict of Interest: The authors declare that the research was conducted in the absence of any commercial or financial relationships that could be construed as a potential conflict of interest.

Copyright $\odot 2021$ Patterson, Byerly and McBride. This is an open-access article distributed under the terms of the Creative Commons Attribution License (CC BY). The use, distribution or reproduction in other forums is permitted, provided the original author(s) and the copyright owner(s) are credited and that the original publication in this journal is cited, in accordance with accepted academic practice. No use, distribution or reproduction is permitted which does not comply with these terms. 